\title{
Investigations on the Plastic Instability in an HCP Mg-Li Alloy
}

\author{
C. Wang, ${ }^{1,2}$ Y. B. Xu, ${ }^{2}$ and E. H. Han' \\ ${ }^{1}$ Environmental Corrosion Center, Institute of Metal Research, Chinese Academy of Sciences, Shenyang 110016, China \\ ${ }^{2}$ Shenyang National Laboratory for Materials Science, Institute of Metal Research, Chinese Academy of Sciences, \\ Shenyang 110016, China \\ Correspondence should be addressed to C. Wang, wangcas@gmail.com
}

Received 24 August 2011; Accepted 30 October 2011

Academic Editor: Michael E. Kassner

Copyright () 2012 C. Wang et al. This is an open access article distributed under the Creative Commons Attribution License, which permits unrestricted use, distribution, and reproduction in any medium, provided the original work is properly cited.

Plastic instability is reported in hexagonal close-packed (HCP) LA41 magnesium alloy during tensile tests. Serration amplitude associated with plastic instability is measured to increase with increasing strain and decrease with increasing strain rate. The model of dynamic strain aging (DSA) is proposed to be the controlling mechanism. Moreover, it is reported that annealing could reduce the flow instability, which has potential practical applications.

\section{Introduction}

Plastic instability associated with the production of PortevinLe Chatelier (PLC) bands, which is also know as serrated flow or jerky flow, is a topic of current theoretical and engineering interest. Lots of studies in this area are on aluminum alloys; however, due to soaring research on magnesium alloys, similar results have been reported in $\mathrm{Mg}-\mathrm{Ag}$ [1], $\mathrm{Mg}-\mathrm{Gd}$ [2], $\mathrm{Mg}$ Y-Nd [3], Mg-Nd [4], and Mg-Al-Zn [5, 6] alloys, respectively.

Microscopically, plastic instability was believed to result from intensive interactions between mobile dislocations and solutes atoms. Cottrell [7] assumed that solute atoms had sufficient mobility to move with mobile dislocations and serrated flow was caused by the drag effect of solute atoms. However, other finding revealed that solute atoms were not expected to have such mobility [8]. Later investigations proposed that the dynamic strain aging (DSA) process during deformation might be the major controlling mechanism [914]. Recently, Picu [15] has suggested a new perspective by considering the effect of solute clusters, which are formed on forest dislocations, on the strength of dislocation junctions to shed more light on this long-lasting yet intriguing issue.

Macroscopic features, such as spatiotemporal localization of plastic deformation and propagation of deformation bands, were also investigated systematically. Experimentally,
Chmelík et al. [16] monitored in situ collective dislocation motion by acoustic emission (AE) and laser extensometry techniques. They concluded that nucleation and propagation of PLC bands were dominated by different dislocation processes. Shabadi et al. [17] and Zhang et al. [18] examined dynamics deformation bands by more sophisticated laser speckle technique. Dablij and Zeghloul [19] characterized the PLC effect by measuring deformation band strains and band propagation velocities. These macroscopic features are also explained theoretically. Particularly, Lebyodkin et al. [20] simulated localized deformation bands patterns by a discrete model based on statistical behavior of stress drops, while Ananthakrishna's extended model [21] explained most of the spatiotemporal features and identified three types of bands. Lebyodkins's mesoscopic model [22], however, pinpointed the dependence of spatiotemporal behavior of strain localizations on deformation conditions.

This paper is intended to report the plastic instability in an HCP magnesium-lithium alloy. The dependence of serration amplitude, which is a major parameter tailoring plastic instability, on strain and strain rate will be discussed in details. Mechanical properties of alloys of the same family will also be compared. It will be shown that plastic instability could be removed through additional annealing treatment, and possible explanations for the disappearance of serrations will also be suggested. 
TABle 1: Chemical compositions of the three alloys under investigation (wt.\%).

\begin{tabular}{lccl}
\hline & $\mathrm{Li}$ & $\mathrm{Al}$ & $\mathrm{Mg}$ \\
\hline LA41 & 4.32 & 0.97 & Balance \\
LA21 & 2.43 & 0.89 & Balance \\
LA40 & 4.06 & - & Balance \\
\hline
\end{tabular}

\section{Experimental Metals}

Three types of Mg-Li alloys, LA41, LA40, and LA21, were investigated in this study. The chemical compositions of the three alloys are listed in Table 1 . The samples were obtained after extrusion with an $8: 1$ ratio at $673 \mathrm{~K}$ and were subsequently homogenized at $623 \mathrm{~K}$ for 1 hour to remove internal stresses. Following that, the samples were quenched in air at room temperature (the as-received state). A batch of LA41 alloy was heat-treated by an extended annealing at $523 \mathrm{~K}$ for 240 hours (the as-annealed state).

Tensile specimens have the dimension of $5 \times 25 \mathrm{~mm}$ by diameter and length, respectively. Uniaxial tensile experiments were performed on an MTS-880 machine at $293 \mathrm{~K}$. Extensometer was attached to gauge length in order to gather accurate strain values. Care was taken to minimize the machine effects. Stress-strain curves were recorded and plotted at a speed of 10 points per second.

The reagent used for microstructure revelation was composed of $3 \mathrm{~g}$ picric acid, $10 \mathrm{~mL}$ acetic acid, $10 \mathrm{~mL}$ distilled water, and $50 \mathrm{~mL}$ ethyl alcohol. Microstructure was characterized by an environmental scanning electron microscope (ESEM, Philips XL30 FEG) under the acceleration voltage of $10 \mathrm{KV}$. Transmission electron microscope (TEM, JEM 2000FXII) samples having diameters of $3 \mathrm{~mm}$ were thinned in a solution of $30 \mathrm{~mL}$ nitric $+70 \mathrm{~mL}$ methanol in a twin jet electropolisher to the final thickness of 50 microns. TEM examinations were conducted at the voltage of $200 \mathrm{KV}$.

\section{Results and Discussion}

3.1. Mechanism for the Onset of Plastic Instability. Figure 1 shows the engineering strain-stress curves of the as-received LA41 at three different strain rates. These curves share similar profiles, with linear-plastic regions, strain-hardening regimes, and stress plateaus. Plastic instability is observed to appear after an incubation strain $\left(\varepsilon_{c}\right)$. It can be seen that the as-received LA41 exhibits a negative strain rate sensitivity (SRS), since the flow stress is continuously lowered with increasing strain rate.

The inset of Figure 1 shows the locally magnified stressstrain curve under the strain rate of $6.66 \times 10^{-4} \mathrm{~s}^{-1}$. The serration amplitude $(\Delta \sigma)$, denoted inside, is defined as the stress difference between the highest point and the lowest one within a serration. Within a serration cycle, a rapid drop follows the gradual stress increase. It should be noted that those high-frequency stress steps are considered as electronic noises because their corresponding amplitude values are generally less than $0.5 \mathrm{MPa}$.

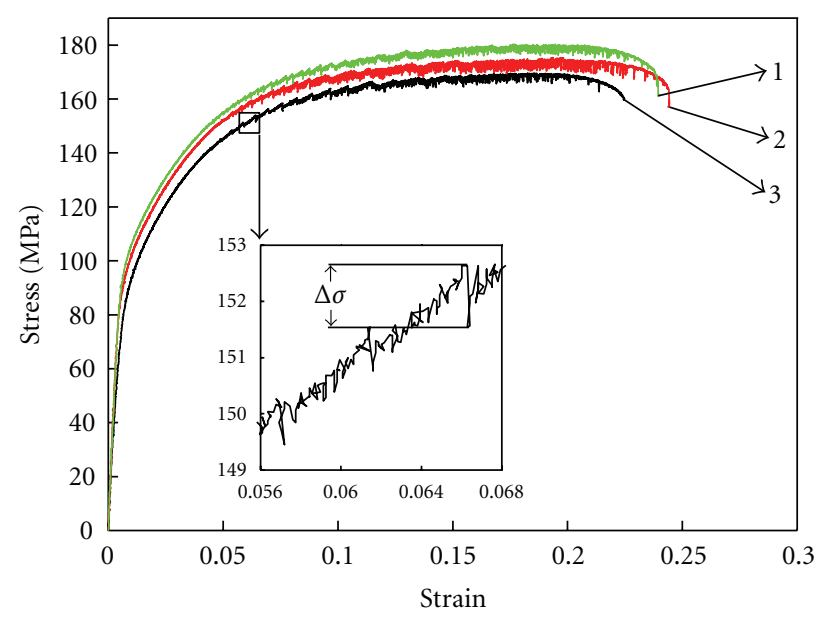

(1) $1.33 * 10^{-4} \mathrm{~s}^{-1}$

(2) $3.33 * 10^{-4} \mathrm{~s}^{-1}$

(3) $6.66 * 10^{-4} \mathrm{~s}^{-1}$

FIGURE 1: Stress-strain curves of the as-received LA41 under different strain rates at $293 \mathrm{~K}$. The inset shows the locally magnified stress-strain curve at the strain rate of $6.66 \times 10^{-4} \mathrm{~s}^{-1}$.

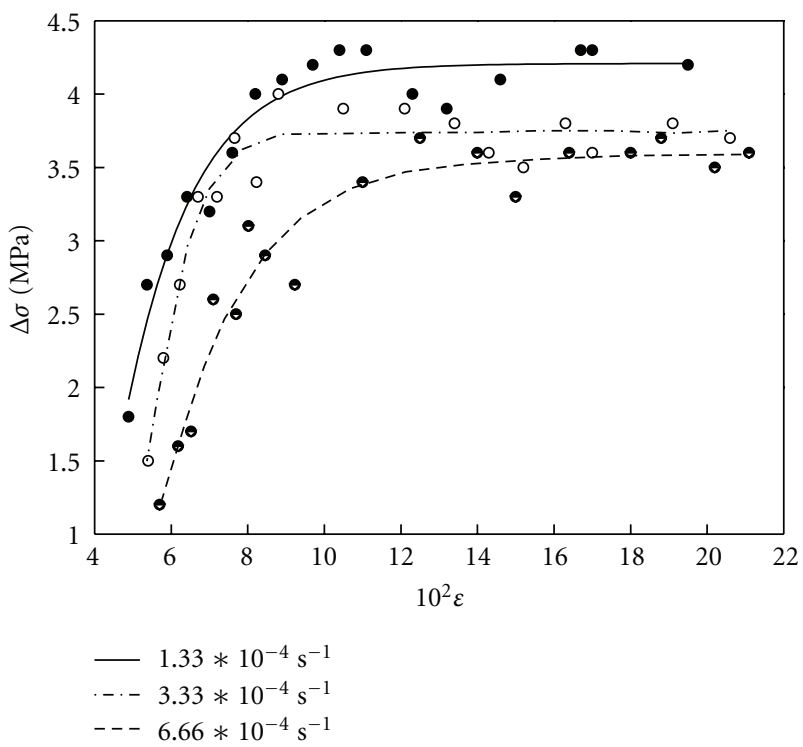

FIGURE 2: Variations of serration amplitude with strain and strain rate of the as-received LA41.

With this definition of serration amplitude, accurate values of $\Delta \sigma$ at various strains under different strain rates were measured by the one-by-one calculation of individual serrations (Figure 2). Through mathematical fitting (corresponding curves), $\Delta \sigma$ is found to increase rapidly at the early stage but get saturated when large strain is achieved. When comparing the influence of the strain rate on serration amplitude, a common trend is that when higher strain rate is high, $\Delta \sigma$ will be decreased to lower values. Summarily, the nature of plastic instability becomes more severe with increasing strain but less severe with increasing strain rate. 


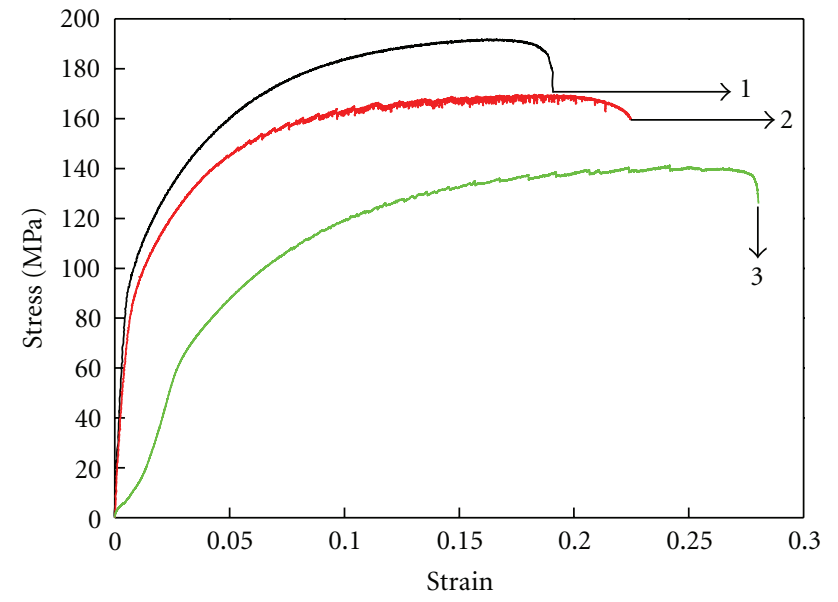

(1) LA21

(2) LA41

(3) LA40

FIGURE 3: Stress-strain curves of three alloys under the strain rate of $6.66 \times 10^{-4} \mathrm{~s}^{-1}$ at $293 \mathrm{~K}$.

Current findings are in good agreement with the characteristics of the DSA model [8, 9, 11, 12, 23-25]. Though DSA is generally a phenomenon observed in dilute alloys, it has been reported to occur in alloys whose solute contents are 20 at.\% or even higher [26]. Existing explanations on the PLC effect of magnesium solid solutions are mainly confined to the framework of classical DSA model $[1-4,6]$. In fact, LA41 itself is a solid solution [27], and, as mentioned above, this alloy shows negative strain rate sensitivity, which is generated via the operation of the dynamic strain aging effect. Therefore, it is justifiable to apply the DSA model to account for plastic instability in LA41, whose total solute content is about 13 at.\%. Diffusion of both $\mathrm{Li}$ and $\mathrm{Al}$ solute atoms is believed to be the fundamental process of dynamic strain aging of the as-received LA41.

Figure 3 shows the comparison of stress-strain curves of the as-received LA41, LA21, and LA40 (Figure 3). These alloys exhibit different ultimate tensile strength (UTS) and yield strength (YS) due to different content of alloying elements of $\mathrm{Al}$ and $\mathrm{Li}$.

It is of great importance to note that serrations are absent in LA21. In a separate paper [28], the activation energy for serrated flow of LA41 is calculated to be $106 \mathrm{KJ} \cdot \mathrm{mol}^{-1}$, which is much larger than diffusion activation energy of $\mathrm{Al}$ and $\mathrm{Li}$, respectively $[29,30]$. That is to say, diffusion of $\mathrm{Al}$ and $\mathrm{Li}$ atoms could have been provoked before the appearance of serrated flow. Therefore, it can be concluded that plastic instability in the as-annealed LA41 is caused by the dynamic strain aging effect through the diffusion of both $\mathrm{Al}$ and $\mathrm{Li}$ atoms, while plastic instability in LA40 is caused by the diffusion of $\mathrm{Li}$ atoms. It could be further inferred that the absence of serrations in LA21 is probably due to insufficient dynamic strain aging effect of solute atoms, especially of $\mathrm{Li}$ atoms.
3.2. Variations of Serration Amplitude. It has been shown that the solute concentration around arrested mobile dislocations is dependent on both strain and strain rate, and the dependence can be described by the following equation [23]:

$$
C=C_{0}\left\{1+\left[\frac{K_{2} \exp \left(-Q_{m} / k T\right) \varepsilon^{m+\beta}}{\dot{\varepsilon}}\right]^{2 / 3}\right\},
$$

where $C$ is the solute concentration around arrested mobile dislocations, $C_{0}$ is the solute concentration in matrix, $K_{2}$ is constant, $Q_{m}$ is the activation energy for the onset of the serrated flow, $m$ and $\beta$ are materials constants related to vacancy concentration and mobile dislocation density, respectively, and $k T$ has its usual meaning. Based on this equation, it is easy to know that the higher the solute concentration around the dislocations, the more intense the interaction between solute atoms and arrested dislocations is, and the larger stress required to overcome obstacles will be. Under a fixed temperature, strain and strain rate are the only two variables. Therefore, from (1), it can be demonstrated that, when the strain rate is fixed, the solute concentration will increase dramatically with increasing strain and $\Delta \sigma$ will be enhanced correspondingly. However, when the strain rate is raised, the diffusing process of solute atoms will possibly be slowed down and the concentration of solute atoms in the vicinity of arrested dislocations will be lowered; therefore, the pinning effect of solute atoms will be appreciably reduced and values $\Delta \sigma$ will be decreased as a corollary.

Another point that needs to be noted is that $\Delta \sigma$ is measured to be constant at large plastic strains. At such strains the deformation may have reached the stage of slow work hardening (stress plateau) where dislocation annihilation and rearrangement become substantial and the rate of generation of vacancies may not be as fast as that of in the rapid work-hardening stage. As a result, the diffusion process of $\mathrm{Al}$ and $\mathrm{Li}$ atoms through vacancies might be reduced, and the concentration of solute atoms around the arrested dislocations will be diluted. Accordingly, the pinning effect of solute atoms could become independent on the strain and the value of $\Delta \sigma$ would remain almost unchanged.

3.3. Disappearance of Serrations. Tensile tests were also performed on the as-annealed LA41 alloy, of which the stressstrain curve is shown in Figure 4 . It can be noticed that the as-annealed LA41 alloy does not exhibit plastic instability. In addition, both YS and UTS decrease a lot after annealing, while the plasticity improves to a certain degree.

Figure 5 shows microstructures of as-received LA41 (Figure 5(a)) and as-annealed LA41 (Figure 5(b)). In the asreceived state, it can be seen that the grains are homogenized and no twins and precipitates are observed. After annealing, however, a large number of lenticular twins are produced, which often end up with sharp points close to grain boundaries (GBs) and appear in multiple parallel groups intersecting each other. Moreover, bright field TEM image of that alloy presents with almost identical information (Figure 6).

The existence of annealing-induced twins might exert significant influence on the as-annealed LA41. It is believed 


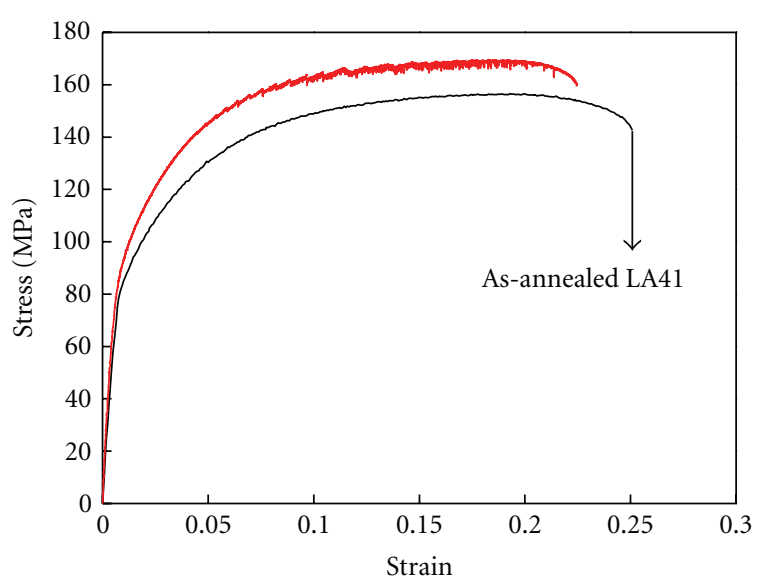

FIGURE 4: Comparison of stress-strain curves of the as-received and the as-annealed LA41 under the strain rate of $6.66 \times 10^{-4} \mathrm{~s}^{-1}$ at $293 \mathrm{~K}$.

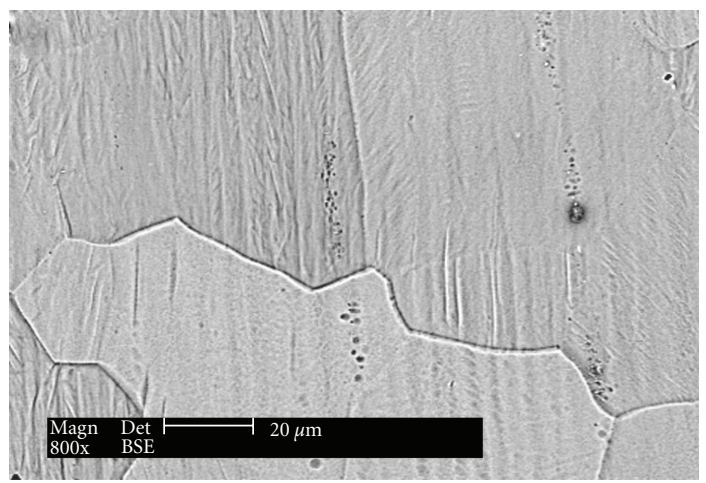

(a)

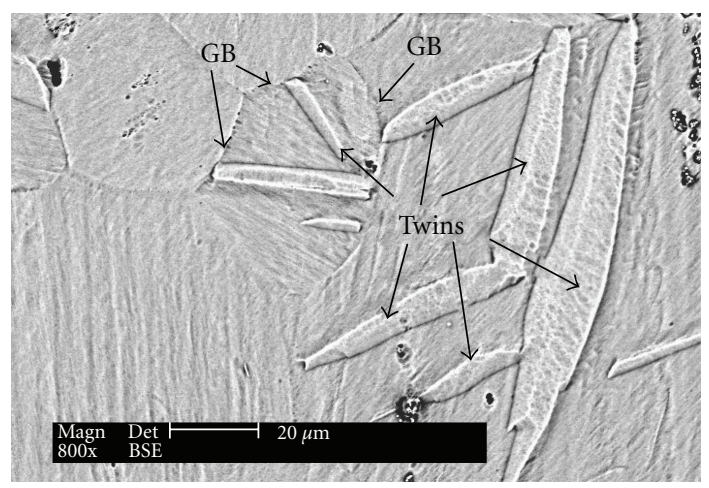

(b)

FIGURE 5: SEM micrographs of (a) the as-received LA41 and (b) the as-annealed LA41.

that they might act as barriers to mobile dislocations, disabling the DSA process to a large extent. Noting that the annealing temperature is well above $0.4 T_{m}\left(T_{m}\right.$ is the melting point) and the annealing time is extremely long, it is important to consider the effect of recrystallization, which would lead to appreciable softening of the alloy and bring about lower YS and UTS as compared to the as-received LA41 alloy.

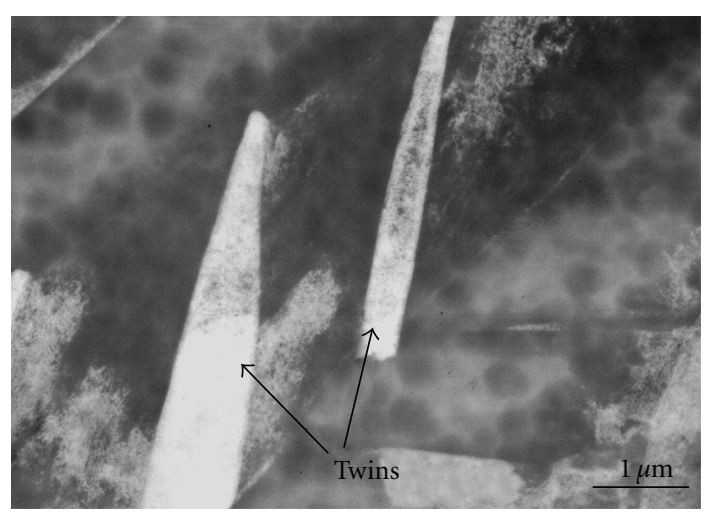

FIGURE 6: Bright field (BF) morphology of the as-annealed LA41.

\section{Conclusion}

Plastic instability was observed in HCP LA41 magnesium alloy in the strain rate range from $1.33 \times 10^{-4} \mathrm{~s}^{-1}$ to $6.66 \times$ $10^{-4} \mathrm{~s}^{-1}$ during tensile tests. Serration amplitude was measured to increase with accumulated strain but decrease with increasing strain rate. The dynamic strain aging model was applied to account for the observed phenomena. It was also demonstrated that additional annealing heat-treatment could remove the plastic instability.

\section{Acknowledgment}

Financial support from National Science Foundation of China (Grant no. 50371089) is greatly acknowledged.

\section{References}

[1] M. Chaturvedi, D. J. Lloyd, and K. Tangri, "Serrated yielding in magnesium-10 wt.-\% Silver alloy," Metal Science, vol. 6, no. 1, pp. 16-19, 1972.

[2] X. Y. Fang, D. Q. Yi, and J. F. Nie, "The serrated flow behavior of Mg-Gd(-Mn-Sc) alloys," Metallurgical and Materials Transactions A, vol. 40, no. 11, pp. 2761-2771, 2009.

[3] S. M. Zhu and J. F. Nie, "Serrated flow and tensile properties of a Mg-Y-Nd alloy," Scripta Materialia, vol. 50, no. 1, pp. 51-55, 2004.

[4] V. Gartnerova, Z. Trojanova, A. Jager, and P. Palcek, "Deformation behaviour of Mg- 0.7 wt.\% Nd alloy," Journal of Alloys and Compounds, vol. 378, no. 1-2, pp. 180-183, 2004.

[5] C. H. Cáceres, C. J. Davidson, J. R. Griffiths, and C. L. Newton, "Effects of solidification rate and ageing on the microstructure and mechanical properties of AZ91 alloy," Materials Science and Engineering A, vol. 325, no. 1-2, pp. 344-355, 2002.

[6] C. Corby, C. H. Cáceres, and P. Lukáč, "Serrated flow in magnesium alloy AZ91," Materials Science and Engineering A, vol. 387-389, no. 1-2, pp. 22-24, 2004.

[7] A. H. Cottrell, "A note on the Portevin-Le Chatelier effect," Philosophical Magazine, vol. 44, no. 355, pp. 829-832, 1953.

[8] P. G. McCormigk, "A model for the Portevin-Le Chatelier effect in substitutional alloys," Acta Metallurgica, vol. 20, no. 3, pp. 351-354, 1972.

[9] A. van den Beukel, "Theory of the effect of dynamic strain aging on mechanical properties," Physica Status Solidi A, vol. 30, no. 1, pp. 197-206, 1975. 
[10] A. W. Sleeswyk, "Slow strain-hardening of ingot iron," Acta Metallurgica, vol. 6, no. 9, pp. 598-603, 1958.

[11] R. A. Mulford and U. F. Kocks, "New observations on the mechanisms of dynamic strain aging and of jerky flow," Acta Metallurgica, vol. 27, no. 7, pp. 1125-1134, 1979.

[12] A. Van Den Beukel, "On the mechanism of serrated yielding and dynamic strain ageing," Acta Metallurgica, vol. 28, no. 7, pp. 965-969, 1980.

[13] P. Penning, "Mathematics of the portevin-le chatelier effect," Acta Metallurgica, vol. 20, no. 10, pp. 1169-1175, 1972.

[14] J. M. Robinson and M. P. Shaw, "Microstructural and mechanical influences on dynamic strain aging phenomena," International Materials Reviews, vol. 39, pp. 113-122, 1994.

[15] R. C. Picu, "A mechanism for the negative strain-rate sensitivity of dilute solid solutions," Acta Materialia, vol. 52, no. 12, pp. 3447-3458, 2004.

[16] F. Chmelík, A. Ziegenbein, H. Neuhäuser, and P. Lukáč, "Investigating the Portevin-Le Chaătelier effect by the acoustic emission and laser extensometry techniques," Materials Science and Engineering A, vol. 324, no. 1-2, pp. 200-207, 2002.

[17] R. Shabadi, S. Kumar, H. J. Roven, and E. S. Dwarakadasa, "Characterisation of PLC band parameters using laser speckle technique," Materials Science and Engineering A, vol. 364, no. 1-2, pp. 140-150, 2004.

[18] Q. Zhang, Z. Jiang, H. Jiang, Z. Chen, and X. Wu, "On the propagation and pulsation of Portevin-Le Chatelier deformation bands: an experimental study with digital speckle pattern metrology," International Journal of Plasticity, vol. 21, no. 11, pp. 2150-2173, 2005.

[19] M. Dablij and A. Zeghloul, "Portevin-Le Chatelier plastic instabilities: characteristics of deformation bands," Materials Science and Engineering A, vol. 237, no. 1, pp. 1-5, 1997.

[20] M. Lebyodkin, Y. Brechet, Y. Estrin, and L. Kubin, "Statistical behaviour and strain localization patterns in the Portevin-Le Chatelier effect," Acta Materialia, vol. 44, no. 11, pp. 45314541, 1996.

[21] G. Ananthakrishna, "Spatio-temporal features of the PortevinLe Chatelier effect," Materials Science and Engineering A, vol. 400-401, no. 1-2, pp. 210-213, 2005.

[22] M. Lebyodkin, L. Dunin-Barkovskii, Y. Bréchet, L. Kubin, and Y. Estrin, "Kinetics and statistics of jerky flow: experiments and computer simulations," Materials Science and Engineering A, vol. 234-236, pp. 115-118, 1997.

[23] C. P. Ling and P. G. McCormick, "The effect of temperature on strain rate sensitivity in an AlMgSi alloy," Acta Metallurgica Et Materialia, vol. 41, no. 11, pp. 3127-3131, 1993.

[24] S. Kumar, "Inverse behaviour of the onset strain of serrated flow," Scripta Metallurgica et Materiala, vol. 33, no. 1, pp. 8186, 1995.

[25] M. Abbadi, P. Hähner, and A. Zeghloul, "On the characteristics of Portevin-Le Chatelier bands in aluminum alloy 5182 under stress-controlled and strain-controlled tensile testing," Materials Science and Engineering A, vol. 337, no. 1-2, pp. 194-201, 2002.

[26] I. S. Kim and M. C. Chaturvedi, "Serrated flow in Al-5 wt.\% Mg alloy," Materials Science and Engineering, vol. 37, no. 2, pp. 165-172, 1979.

[27] D. W. Levinson and D. J. McPherson, "Phase relations in magnesium-lithium-aluminum alloys," Transactions of the American Society for Metals, vol. 48, pp. 689-705, 1956.

[28] C. Wang, Y. Xu, and E. Han, "PLC effect and its explanation of LA41 magnesium alloys," Acta Metallurgica Sinica, vol. 42, no. 2, pp. 191-194, 2006.
[29] W. Gasior and Z. Moser, "Chemical diffusion coefficients in solid al-rich aluminium-magnesium alloys," Archives of Metallurgy, vol. 46, no. 4, pp. 455-463, 2001.

[30] W. Gasior and Z. Moser, Archives of Metallurgy, vol. 46, p. 269, 2001. 

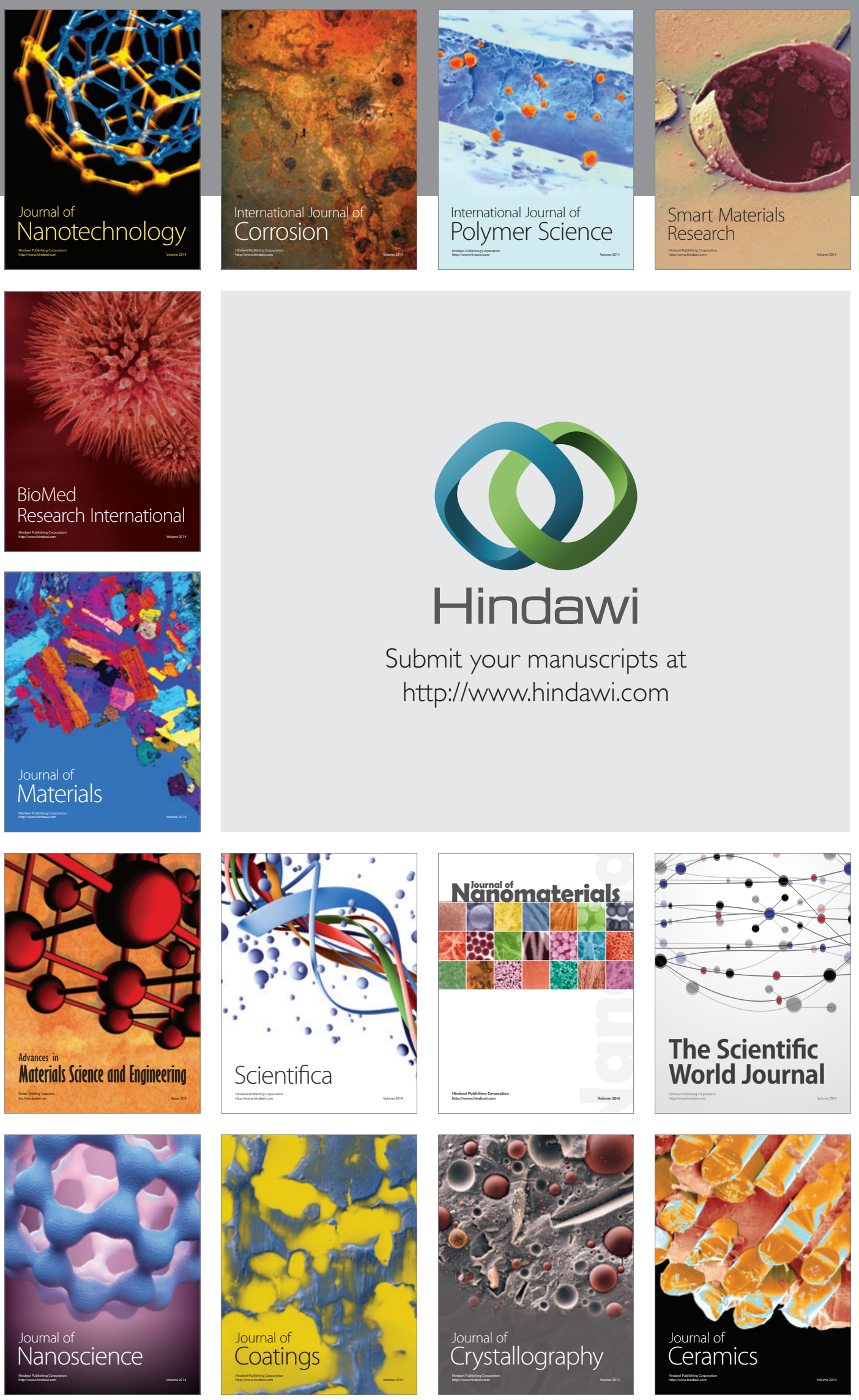

The Scientific World Journal

Submit your manuscripts at

http://www.hindawi.com

\section{World Journal}

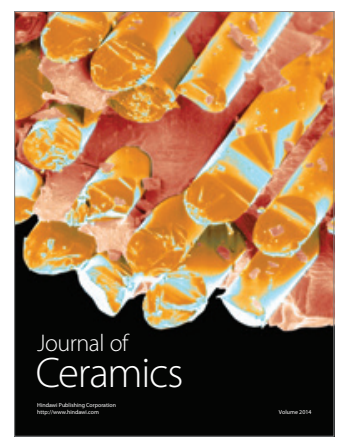

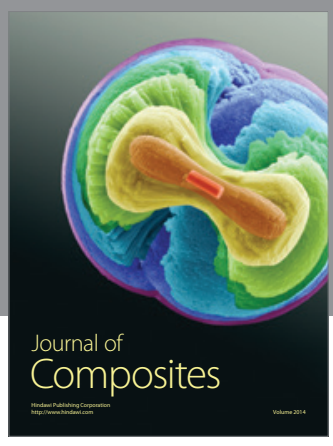
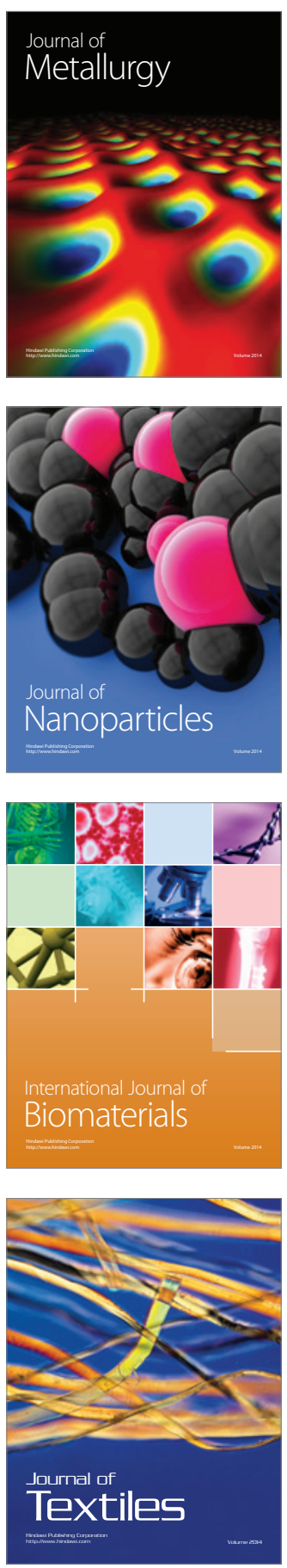